\title{
REDUCED-ORDER FRACTIONAL DESCRIPTOR OBSERVERS FOR A CLASS OF FRACTIONAL DESCRIPTOR CONTINUOUS-TIME NONLINEAR SYSTEMS
}

\author{
TADEUSZ KACZOREK ${ }^{a}$ \\ ${ }^{a}$ Faculty of Electrical Engineering \\ Białystok University of Technology, ul. Wiejska 45D, 15-351 Białystok, Poland \\ e-mail: kaczorek@isep.pw.edu.pl
}

\begin{abstract}
Fractional descriptor reduced-order nonlinear observers for a class of fractional descriptor continuous-time nonlinear systems are proposed. Sufficient conditions for the existence of the observers are established. The design procedure for the observers is given and demonstrated on a numerical example.
\end{abstract}

Keywords: fractional system, descriptor system, nonlinear systems, reduced-order observer.

\section{Introduction}

Fractional linear systems were considered in many papers and books (Kaczorek, 2012b; 2013; 2011a; 2011b; Oldham and Spanier, 1974; Ostalczyk, 2008; Podlubny, 1999; Vinagre et al., 2002). Positive linear systems consisting of $n$ subsystems with different fractional orders were proposed by Kaczorek (2011a; 2011b). Descriptor (singular) linear systems were investigated by Dodig and Stosic (2009), Cuihong (2012), Dai (1989), Fahmy and O’Reill (1989), Gantmacher (1960), Guang-ren (2010), Kaczorek (2012a; 2012b; 1992), Kucera and Zagalak (1988), Lewis (1983), Luenberger (1978; 1977), Podlubny (1999) and Van Dooren (1979). Eigenvalue and invariant assignments by state and input feedbacks were addressed by Fahmy and O'Reill (1989), as well as Kaczorek (2004; 2015). The computation of Kronecker's canonical form of a singular pencil was analyzed by Van Dooren (1979).

A new concept of perfect observers for linear continuous-time systems was proposed by Kaczorek (2001; 2015). Observers for fractional linear systems were addressed by Kaczorek (2008), Kociszewski (2013) and Vinagre et al. (2002), and for descriptor linear systems by Kaczorek (2014a). Fractional descriptor full-order observers for fractional descriptor continuous-time linear systems were proposed by Kaczorek (2001), along with reduced-order observers (Kaczorek, 2014b). The stability of positive descriptor systems was investigated by Virnik (2008).
In this paper, fractional descriptor reduced-order observers for a class of fractional descriptor continuous-time nonlinear systems will be proposed and sufficient conditions for the existence of the observer will be established.

The paper is organized as follows. In Section 2, the basic definitions and theorems of fractional descriptor nonlinear continuous-time systems are recalled and their full-order fractional descriptor observers are presented. In Section 3, reduced-order fractional descriptor nonlinear observers are proposed and sufficient conditions for the existence for observers are established. A design procedure of the reduced-order observers and an illustrating example are given in Section 4. Concluding remarks are presented in Section 5.

\section{Fractional descriptor systems and their full-order observers}

Consider the fractional descriptor continuous-time linear system

$$
\begin{aligned}
E \frac{\mathrm{d}^{\alpha} x}{\mathrm{~d} t^{\alpha}} & =A x+B u, \quad x_{0}=x(0), \\
y & =C x,
\end{aligned}
$$


where $\mathrm{d}^{\alpha} x / \mathrm{d} t^{\alpha}$ is the fractional $\alpha$-order derivative defined by Caputo,

$$
\begin{array}{r}
{ }_{0} D_{t}^{\alpha} x(t) \\
=\frac{\mathrm{d}^{\alpha} x(t)}{\mathrm{d} t^{\alpha}}=\frac{1}{\Gamma(n-\alpha)} \int_{0}^{t} \frac{\frac{\mathrm{d}^{n} x}{\mathrm{~d} \tau^{n}}}{(t-\tau)^{\alpha-n+1}} \mathrm{~d} \tau, \\
n-1<\alpha<n \in \mathbb{N},
\end{array}
$$

and

$$
\Gamma(x)=\int_{0}^{\infty} e^{-t} t^{x-1} \mathrm{~d} t
$$

is the gamma function, while $x=x(t) \in \mathbb{R}^{n}, u=u(t) \in$ $\mathbb{R}^{m}, y=y(t) \in \mathbb{R}^{p}$ are the state, input and output vectors, $E, A \in \mathbb{R}^{n \times n}, B \in \mathbb{R}^{n \times m}, C \in \mathbb{R}^{p \times n}$. It is assumed that $\operatorname{det} E=0$ and

$$
\operatorname{det}[E \lambda-A] \neq 0
$$

for some $\lambda \in \mathbb{C}$.

Let $U$ be the set of admissible inputs $u(t) \in U \subset \mathbb{R}^{m}$ and $X_{0} \subset \mathbb{R}^{n}$ be the set of consistent initial conditions $x_{0} \in X_{0}$ for which Eqn. (1) has a solution $x(t)$ for $u(t) \in$ $U$.

The solution of Eqn. (1a) for $x_{0} \in X_{0}$ has been derived by Kaczorek (2014a).

Definition 1. The fractional descriptor linear system (1) is called asymptotically stable if $\lim _{t \rightarrow \infty} x(t)=0$ for any finite $x_{0} \in X_{0}$ and $u(t)=0$.

Theorem 1. (Kaczorek, 2011b; Matignon, 1996) The fractional descriptor linear system (1) is asymptotically stable if and only if the zeros (the eigenvalues of $(E, A)$ ) $\lambda_{1}, \ldots, \lambda_{p}$ of the equation

$$
\operatorname{det}[E \lambda-A]=\lambda^{p}+a_{p-1} \lambda^{p-1}+\cdots+a_{1} \lambda+a_{0}=0
$$

satisfy the condition

$$
\left|\arg \lambda_{k}\right|>\alpha \frac{\pi}{2}
$$

for $k=1, \ldots, p$.

The eigenvalues satisfying the condition (5) are located in the stability region shown in Fig. 1 and denoted by $S_{r}$.

Definition 2. The fractional descriptor continuous-time linear system

$$
E \frac{\mathrm{d}^{\alpha} \hat{x}}{\mathrm{~d} t^{\alpha}}=F \hat{x}+G u+H y
$$

where $\hat{x}=\hat{x}(t) \in \mathbb{R}^{n}$ is the estimate of $x(t)$, and $u=$ $u(t) \in \mathbb{R}^{m}, y=y(t) \in \mathbb{R}^{p}$ are the same input and output vectors as in (1), $E, F \in \mathbb{R}^{n \times n}, G \in \mathbb{R}^{n \times m}, H \in \mathbb{R}^{n \times p}$, $\operatorname{det} E=0$ is called a (full-order) state observer for the system (1) if

$$
\lim _{t \rightarrow \infty}[x(t)-\hat{x}(t)]=0 .
$$

Theorem 2. (Guang-ren, 2010; Kaczorek, 1992) The fractional descriptor system (1) has a full state observer (6) if and only if there exists a matrix $H$ such that all eigenvalues of the pair $(E, A-H C)$ are located in the stable region $S_{r}$ shown in Fig. 1, i.e.,

$$
\sigma(E, A-H C) \subset S_{r},
$$

where $\sigma$ denotes the spectrum of the pair.

The proof is also given by Kaczorek (2014a).

From Theorem 1 it follows that the design of a stable observer (6) of the system (1) has been reduced to finding a matrix $\mathrm{H}$ such that the eigenvalues of the pair $(E, A-H C)$ are located in the asymptotic stability region. It is well-known (Guang-ren, 2010; Kaczorek, 1992) that there exists a matrix $H$ such that the eigenvalues of the pair $(E, A-H C)$ are located in the asymptotic stability region if and only if the fractional descriptor system (1) is detectable (Guang-ren, 2010; Kaczorek, 1992), i.e.,

$$
\operatorname{rank}\left[\begin{array}{c}
E s_{k}-A \\
C
\end{array}\right]=n
$$

for $s_{k} \in \sigma(E, A)$.

The problem of designing the observer (6) of the system (1) can be reduced to the procedure of designing a state-feedback of the form $v=-H^{T} x$ for the dual system (Guang-ren, 2010; Kaczorek, 1992)

$$
E^{T} \frac{\mathrm{d}^{\alpha} x}{\mathrm{~d} t^{\alpha}}=A^{T} x+C^{T} v .
$$

To guarantee that the descriptor state observer is impulse-free, the matrix $H$ must be chosen so that

$$
\operatorname{deg}[\operatorname{det}(E s-A+H C)]=\operatorname{rank} E .
$$

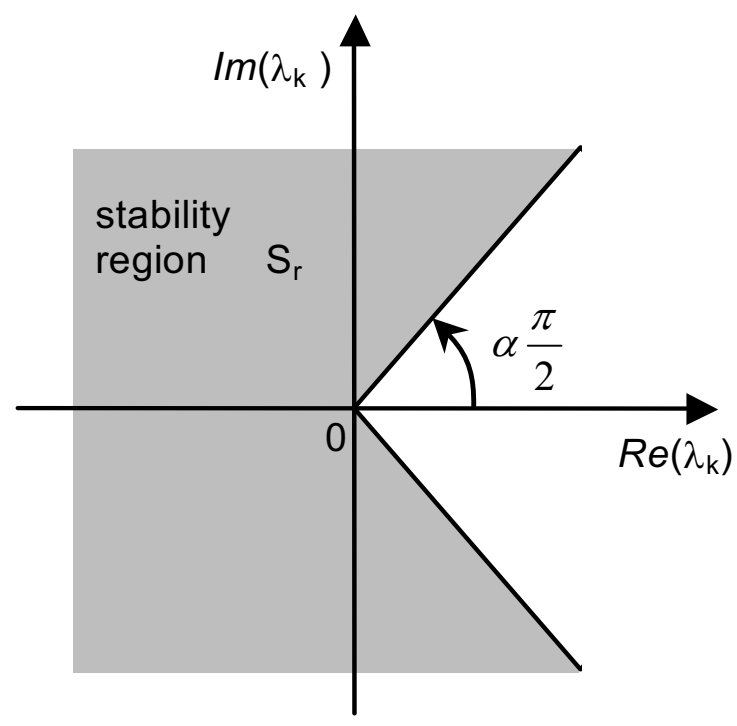

Fig. 1. Stability region. 
It is well known (Cuihong, 2012; Kaczorek, 1992) that the finite observers poles (the finite eigenvalues of the pair $(E, A-H C)$ can be arbitrarily assigned if and only if the descriptor system (1) is R-observable, i.e.,

$$
\operatorname{rank}\left[\begin{array}{c}
E s-A \\
C
\end{array}\right]=n
$$

for $s \in \mathbb{C}$

Therefore, the following theorem has been proved.

Theorem 3. An impulse-free fractional descriptor observer (6) with an arbitrary prescribed set of poles of the fractional descriptor system (1) satisfying (3) if and only exists if the conditions (11) and (12) are met.

Now let us consider the fractional descriptor continuous-time nonlinear system

$$
\begin{aligned}
E \frac{\mathrm{d}^{\alpha} x}{\mathrm{~d} t^{\alpha}} & =A x+f(x, u), \quad x_{0}=x(0), \\
y & =C x,
\end{aligned}
$$

where $x=x(t) \in \mathbb{R}^{n}, u=u(t) \in \mathbb{R}^{m}, y=y(t) \in \mathbb{R}^{p}$ are respectively the state, input and output vectors, $E, A \in$ $\mathbb{R}^{n \times n}, f(x, u) \in \mathbb{R}^{n}$ is a continuous vector function of $x$ and $u$.

It is assumed that $\operatorname{det} E=0$ and (3) is met.

Definition 3. The fractional descriptor continuous-time nonlinear system

$$
E \frac{\mathrm{d}^{\alpha} \hat{x}}{\mathrm{~d} t^{\alpha}}=F \hat{x}+f(x, u)+H y
$$

is called a full-order observer of the nonlinear system (13) if

$$
\lim _{t \rightarrow \infty}[x(t)-\hat{x}(t)]=0,
$$

where $\hat{x}=\hat{x}(t) \in \mathbb{R}^{n}$ is the estimate of $x \in \mathbb{R}^{n}, u \in \mathbb{R}^{m}$ and $f(x, u), y \in \mathbb{R}^{p}$ are the same vectors as in (13).

Definition 4. The fractional descriptor nonlinear system (14) is called a full-order perfect observer of the nonlinear system (13) if

$$
x(t)=\hat{x}(t) \text { for } \quad t>0 .
$$

A design method of full-order perfect observers of nonlinear systems has been proposed by Kaczorek (2015).

\section{Reduced-order fractional descriptor nonlinear observers}

Consider the fractional descriptor nonlinear system (13) satisfying the assumption (3).

If

$$
\operatorname{rank} C=p \text {, }
$$

then there exist a permutation matrix $P \in \mathbb{R}^{n \times n}$

$$
\begin{aligned}
& C P=\left[\begin{array}{ll}
C_{1} & C_{2}
\end{array}\right], \quad C_{1} \in \mathbb{R}^{p \times p}, \\
& \operatorname{det} C_{1} \neq 0 \text {, } \\
& C_{2} \in \mathbb{R}^{p \times(n-p)}
\end{aligned}
$$

and the nonsingular matrix

$$
Q_{1}=\left[\begin{array}{cc}
C_{1}^{-1} & -C_{1}^{-1} C_{2} \\
0 & I_{n-p}
\end{array}\right] \in \mathbb{R}^{n \times n}
$$

such that

$$
\begin{aligned}
\bar{C} & =C P Q_{1} \\
& =\left[\begin{array}{ll}
C_{1} & C_{2}
\end{array}\right] Q_{1} \\
& =\left[\begin{array}{ll}
C_{1} & C_{2}
\end{array}\right]\left[\begin{array}{cc}
C_{1}^{-1} & -C_{1}^{-1} C_{2} \\
0 & I_{n-p}
\end{array}\right]=\left[\begin{array}{ll}
I_{p} & 0
\end{array}\right] .
\end{aligned}
$$

Substituting

$$
x=P Q_{1} \bar{x}
$$

into (13), we obtain

$$
\begin{aligned}
& E P Q_{1} \frac{\mathrm{d}^{\alpha} \bar{x}}{\mathrm{~d} t^{\alpha}}=A P Q_{1} \bar{x}+\left.f(x, u)\right|_{x=P Q_{1} \bar{x}}, \\
& y=C x=C P Q_{1} \bar{x}=\left[\begin{array}{ll}
I_{p} & 0
\end{array}\right]\left[\begin{array}{l}
\bar{x}_{1} \\
\bar{x}_{2}
\end{array}\right]=\bar{x}_{1}, \\
& \bar{x}_{1} \in \mathbb{R}^{p}, \quad \bar{x}_{2} \in \mathbb{R}^{(n-p)} .
\end{aligned}
$$

Premultiplying (22a) by a nonsingular elementary row operations matrix $Q_{2} \in \mathbb{R}^{n \times n}$, we obtain

$$
\begin{aligned}
Q_{2} E P Q_{1} & =\left[\begin{array}{cc}
E_{11} & 0 \\
E_{21} & E_{22}
\end{array}\right], \quad E_{11} \in \mathbb{R}^{p \times p}, \\
E_{21} & \in \mathbb{R}^{(n-p) \times p}, \quad E_{22} \in \mathbb{R}^{(n-p) \times(n-p)}
\end{aligned}
$$

and

$$
\begin{aligned}
& E_{11} \frac{\mathrm{d}^{\alpha} \bar{x}_{1}}{\mathrm{~d} t^{\alpha}}=A_{11} \bar{x}_{1}+A_{12} \bar{x}_{2}+f_{1}\left(\bar{x}_{1}, u\right), \\
& E_{21} \frac{\mathrm{d}^{\alpha} \bar{x}_{1}}{\mathrm{~d} t^{\alpha}}+E_{22} \frac{\mathrm{d}^{\alpha} \bar{x}_{2}}{\mathrm{~d} t^{\alpha}}=A_{21} \bar{x}_{1}+A_{22} \bar{x}_{2} \\
& +f_{2}\left(\bar{x}_{1}, \bar{x}_{2}, u\right),
\end{aligned}
$$

where

$$
\begin{aligned}
& Q_{2} A P Q_{1}=\left[\begin{array}{ll}
A_{11} & A_{12} \\
A_{21} & A_{22}
\end{array}\right], \\
& A_{11} \in \mathbb{R}^{p \times p}, \quad A_{12} \in \mathbb{R}^{p \times(n-p)}, \\
& A_{21} \in \mathbb{R}^{(n-p) \times n}, \quad A_{22} \in \mathbb{R}^{(n-p) \times(n-p)}, \\
& {\left[\begin{array}{c}
f_{1}\left(\bar{x}_{1}, u\right) \\
f_{2}\left(\bar{x}_{1}, \bar{x}_{2}, u\right)
\end{array}\right]=Q_{2} f(P Q \bar{x}, u),} \\
& f_{1}\left(\bar{x}_{1}, u\right) \in \mathbb{R}^{p}, \quad f_{2}\left(\bar{x}_{1}, \bar{x}_{2}, u\right) \in \mathbb{R}^{n-p} .
\end{aligned}
$$

From (22b) it follows that $\bar{x}_{1}=y$, and for given $y$ the subvector $\bar{x}_{1}$ is known. Therefore, the reduced-order 
observer of the fractional descriptor system (1) should reconstruct only the subvector $\bar{x}_{2}=\mathbb{R}^{(n-p)}$.

From (24) we have

$$
\begin{aligned}
E_{22} \frac{\mathrm{d}^{\alpha} \bar{x}_{2}}{\mathrm{~d} t^{\alpha}} & =A_{22} \bar{x}_{2}+\bar{f}_{2}\left(\bar{x}_{1}, \bar{x}_{2}, u\right), \\
\bar{y} & =A_{12} \bar{x}_{2},
\end{aligned}
$$

where

$$
\bar{f}_{2}\left(\bar{x}_{1}, \bar{x}_{2}, u\right)=f_{2}\left(\bar{x}_{1}, \bar{x}_{2}, u\right)-E_{21} \frac{\mathrm{d}^{\alpha} y}{\mathrm{~d} t^{\alpha}}+A_{21} y
$$

and

$$
\bar{y}=E_{11} \frac{\mathrm{d}^{\alpha} y}{\mathrm{~d} t^{\alpha}}-A_{11} y-f_{2}\left(\bar{x}_{1}, \bar{x}_{2}, u\right)
$$

are the new known input and output, respectively.

To find the estimate $\hat{x}_{2}$ of $\bar{x}_{2}$, the following full-order fractional descriptor nonlinear observer for the system (25) can be applied (Kaczorek, 2014a).

Definition 5. The fractional descriptor continuous-time nonlinear system

$$
E_{22} \frac{\mathrm{d}^{\alpha} \hat{x}_{2}}{\mathrm{~d} t^{\alpha}}=F \hat{x}_{2}+\bar{f}_{2}\left(\bar{x}_{1}, \bar{x}_{2}, u\right)+H \bar{y}
$$

where $\hat{x}_{2} \in \mathbb{R}^{n-p}, F \in \mathbb{R}^{(n-p) \times(n-p)}, H \in \mathbb{R}^{(n-p) \times p}$, is called a reduced-order fractional descriptor observer for the nonlinear system (13) if

$$
\lim _{t \leftarrow \infty}\left[\bar{x}_{2}(t)-\hat{x}_{2}(t)\right]=0 .
$$

Applying Theorem 1 to the fractional descriptor system (25), we obtain the following result.

Theorem 4. For the fractional descriptor nonlinear system (13) the reduced-order observer (26) exists if the system (25) is detectable, i.e.,

$$
\operatorname{rank}\left[\begin{array}{c}
E_{22} s-A_{22} \\
A_{12}
\end{array}\right]=n-p
$$

for $s_{k} \in \sigma\left(E_{22}, A_{22}\right)$.

It is well known (Guang-ren, 2010) that the eigenvalues of $\left(E_{22}, A_{22}\right)$ (the finite poles of the observer) can be arbitrarily assigned if and only if the descriptor system (25) is $R$-observable, i.e.,

$$
\operatorname{rank}\left[\begin{array}{c}
E_{22} s-A_{22} \\
A_{12}
\end{array}\right]=n-p
$$

for all $s \in \mathbb{C}$.

To guarantee that the descriptor observer (26) is impulse-free, the matrix $H$ should be chosen so that (Guang-ren, 2010)

$$
\operatorname{deg}\left\{\operatorname{det}\left[E_{22} s-A_{22}+H A_{12}\right]\right\}=\operatorname{rank} E_{22} .
$$

Therefore, the following theorem has been proved.
Theorem 5. The impulse-free reduced-order observer (26) for the fractional descriptor system (13) satisfying (3) exists if the conditions (24c), (29) and (30) are met.

Remark 1. If $E_{22}=0$ and $\operatorname{det} A_{22} \neq 0$, then from (25a) we have

$$
\bar{x}_{2}=-A_{22}^{-1} \bar{f}_{2}\left(\bar{x}_{1}, \bar{x}_{2}, u\right),
$$

and we can find $\bar{x}_{2}$ without any observer.

Remark 2. If $\operatorname{det} E_{22} \neq 0$, then from (25a) we have

$$
\frac{\mathrm{d}^{\alpha} \bar{x}_{2}}{\mathrm{~d} t^{\alpha}}=E_{22}^{-1} A_{22} \bar{x}_{2}+E_{22}^{-1} \bar{f}_{2}\left(\bar{x}_{1}, \bar{x}_{2}, u\right),
$$

and the estimate $\hat{x}_{2}$ of $\bar{x}_{2}$ can be found using the classical (standard) fractional observer (Kociszewski, 2013; Kaczorek, 2004).

\section{Procedure and examples}

To design the reduced-order observer (26) for the fractional descriptor nonlinear system (13), the following procedure can be used.

\section{Procedure 1.}

Step 1. Find a permutation matrix $P$ and a nonsingular matrix (19) transferring the matrix $C$ to the form (20).

Step 2. Find the elementary row operations matrix $Q_{2}$, and using (23) and (24c) compute the matrices $E_{11}, E_{21}, E_{22}$, $A_{11}, A_{12}, A_{21}, A_{22}$, and $f_{2}\left(\bar{x}_{1}, u\right), f_{2}\left(\bar{x}_{1}, \bar{x}_{2}, u\right)$.

Step 3. Check the conditions (28) and (30) for some $H \in$ $\mathbb{R}^{(n-p) \times p}$.

Step 4. Using

$$
F=A_{22}-H A_{12},
$$

find a matrix $H$ such that the pair $\left(E_{22}, F\right)$ has the desired eigenvalues located in the stability region $S_{r}$.

Step 5. Find Eqn. 26 of the desired fractional descriptor nonlinear observer.

Example 1. Consider the fractional descriptor system (13) with

$$
\begin{aligned}
& E=\left[\begin{array}{llll}
1 & 0 & 0 & 0 \\
0 & 0 & 0 & 0 \\
0 & 0 & 0 & 1 \\
0 & 0 & 1 & 0
\end{array}\right] \\
& A=\left[\begin{array}{cccc}
-1 & 0 & 2 & 1 \\
0 & -2 & 0 & 0 \\
0 & 0 & 1 & 0 \\
0 & 0 & 0 & 1
\end{array}\right] \\
& f(x, u)=\left[\begin{array}{c}
x_{2}^{2}+u^{2} \\
x_{2} x_{4}+2 u \\
x_{1} x_{2}+x_{3}^{2}+x_{2} u \\
x_{2} x_{3}+2 x_{1} u^{2}
\end{array}\right] \text {, } \\
& C=\left[\begin{array}{llll}
0 & 1 & 0 & 1 \\
1 & 0 & 0 & 0
\end{array}\right] \text {. }
\end{aligned}
$$


The system satisfies the condition (3) since

$$
\begin{aligned}
\operatorname{det}[E s-A] & =\left[\begin{array}{cccc}
s+1 & 0 & -2 & -1 \\
0 & 2 & 0 & 0 \\
0 & 0 & -1 & s \\
0 & 0 & s & -1
\end{array}\right] \\
& =2(s+1)\left(1-s^{2}\right) \neq 0 .
\end{aligned}
$$

Using Procedure 1, we obtain what follows.

Step 1. In this case the permutation matrix is $P=I_{4}$ (the identity matrix)

$$
\begin{aligned}
C P & =\left[\begin{array}{cc}
C_{1} & C_{2}
\end{array}\right], \\
C_{1} & =\left[\begin{array}{ll}
0 & 1 \\
1 & 0
\end{array}\right], \quad C_{2}=\left[\begin{array}{ll}
0 & 1 \\
0 & 0
\end{array}\right], \\
Q_{1} & =\left[\begin{array}{cc}
C_{1}^{-1} & -C_{1}^{-1} C_{2} \\
0 & I_{n-p}
\end{array}\right] \\
& =\left[\begin{array}{cccc}
0 & 1 & 0 & 0 \\
1 & 0 & 0 & -1 \\
0 & 0 & 1 & 0 \\
0 & 0 & 0 & 1
\end{array}\right]
\end{aligned}
$$

and

$$
\begin{aligned}
\bar{C} & =C P Q_{1}=\left[\begin{array}{llll}
0 & 1 & 0 & 1 \\
1 & 0 & 0 & 0
\end{array}\right]\left[\begin{array}{cccc}
0 & 1 & 0 & 0 \\
1 & 0 & 0 & -1 \\
0 & 0 & 1 & 0 \\
0 & 0 & 0 & 1
\end{array}\right] \\
& =\left[\begin{array}{llll}
1 & 0 & 0 & 0 \\
0 & 1 & 0 & 0
\end{array}\right] .
\end{aligned}
$$

The new state vector is given by

$$
\begin{aligned}
\bar{x} & =P^{-1} Q_{1}^{-1} x=\left[\begin{array}{llll}
0 & 1 & 0 & 1 \\
1 & 0 & 0 & 0 \\
0 & 0 & 1 & 0 \\
0 & 0 & 0 & 1
\end{array}\right]\left[\begin{array}{l}
x_{1} \\
x_{2} \\
x_{3} \\
x_{4}
\end{array}\right] \\
& =\left[\begin{array}{c}
x_{2}+x_{4} \\
x_{1} \\
x_{3} \\
x_{4}
\end{array}\right]=\left[\begin{array}{l}
\bar{x}_{1} \\
\bar{x}_{2}
\end{array}\right], \\
\bar{x}_{1} & =\left[\begin{array}{c}
x_{2}+x_{4} \\
x_{1}
\end{array}\right], \quad \bar{x}_{2}=\left[\begin{array}{l}
x_{3} \\
x_{4}
\end{array}\right] .
\end{aligned}
$$

Step 2. The matrix of elementary operations is equal to $Q_{2}=I_{4}$ and

$$
\begin{aligned}
E Q_{1}= & {\left[\begin{array}{llll}
1 & 0 & 0 & 0 \\
0 & 0 & 0 & 0 \\
0 & 0 & 0 & 1 \\
0 & 0 & 1 & 0
\end{array}\right]\left[\begin{array}{cccc}
0 & 1 & 0 & 0 \\
1 & 0 & 0 & -1 \\
0 & 0 & 1 & 0 \\
0 & 0 & 0 & 1
\end{array}\right] } \\
& =\left[\begin{array}{llll}
0 & 1 & 0 & 0 \\
0 & 0 & 0 & 0 \\
0 & 0 & 0 & 1 \\
0 & 0 & 1 & 0
\end{array}\right]=\left[\begin{array}{cc}
E_{11} & 0 \\
0 & E_{22}
\end{array}\right],
\end{aligned}
$$

$$
\begin{aligned}
& E_{11}=\left[\begin{array}{ll}
0 & 1 \\
0 & 0
\end{array}\right], \quad E_{22}=\left[\begin{array}{ll}
0 & 1 \\
1 & 0
\end{array}\right], \\
& A Q_{1}=\left[\begin{array}{cccc}
-1 & 0 & 2 & 1 \\
0 & -2 & 0 & 0 \\
0 & 0 & 1 & 0 \\
0 & 0 & 0 & 1
\end{array}\right]\left[\begin{array}{cccc}
0 & 1 & 0 & 0 \\
1 & 0 & 0 & -1 \\
0 & 0 & 1 & 0 \\
0 & 0 & 0 & 1
\end{array}\right] \\
& =\left[\begin{array}{cccc}
0 & -1 & 2 & 1 \\
-2 & 0 & 0 & 2 \\
0 & 0 & 1 & 0 \\
0 & 0 & 0 & 1
\end{array}\right]=\left[\begin{array}{cc}
A_{11} & A_{12} \\
A_{21} & A_{22}
\end{array}\right] \text {, } \\
& A_{11}=\left[\begin{array}{cc}
0 & -1 \\
-2 & 0
\end{array}\right], \quad A_{12}=\left[\begin{array}{ll}
2 & 1 \\
0 & 2
\end{array}\right], \\
& A_{21}=\left[\begin{array}{ll}
0 & 0 \\
0 & 0
\end{array}\right], \quad A_{22}=\left[\begin{array}{ll}
1 & 0 \\
0 & 1
\end{array}\right] \text {, } \\
& {\left[\begin{array}{c}
f_{1}\left(\bar{x}_{1}, u\right) \\
f_{2}\left(\bar{x}_{1}, \bar{x}_{2}, u\right)
\end{array}\right]=f(x, u) .}
\end{aligned}
$$

Step 3. The conditions 28 and (30) are satisfied since

$$
\operatorname{rank}\left[\begin{array}{c}
E_{22} s-A_{22} \\
A_{12}
\end{array}\right]=\operatorname{rank}\left[\begin{array}{cc}
-1 & s \\
s & -1 \\
2 & 1 \\
0 & 2
\end{array}\right]
$$$$
=2
$$

for all $s \in \mathbb{C}$, and for

$$
H=\left[\begin{array}{ll}
h_{11} & h_{12} \\
h_{21} & h_{22}
\end{array}\right]
$$

we have

$$
\begin{aligned}
& \operatorname{deg}\left\{\operatorname{det}\left[E_{22} s-A_{22}+H A_{12}\right]\right\} \\
& \quad=\operatorname{deg}\left\{\operatorname{det}\left[\begin{array}{cc}
-1+2 h_{11} & s+h_{11}+2 h_{12} \\
s+2 h_{21} & -1+h_{21}+2 h_{22}
\end{array}\right]\right\} \\
& \quad=2=\operatorname{rank} E_{2} .
\end{aligned}
$$

Step 4. Using (33) we obtain

$$
F=A_{22}-H A_{12}=\left[\begin{array}{cc}
1-2 h_{11} & -2 h_{12}-h_{11} \\
-2 h_{21} & 1-2 h_{22}-h_{21}
\end{array}\right] \text {. }
$$

Let the desired eigenvalues of the pair $\left(E_{22}, F\right)$ be $s_{d 1}=s_{d 2}=-10$. Then

$$
\begin{aligned}
\operatorname{det} & {\left[E_{22} s-F\right] } \\
= & \left|\begin{array}{cc}
2 h_{11}-1 & s+h_{11}+2 h_{12} \\
s+2 h_{21} & h_{21}+2 h_{22}-1
\end{array}\right| \\
= & -s^{2}-\left(2 h_{21}+h_{11}+2 h_{12}\right) s \\
& +\left(2 h_{11}-1\right)\left(h_{21}+2 h_{22}-1\right) \\
& -2 h_{21}\left(h_{11}+2 h_{12}\right) \\
= & -(s+10)^{2}=-\left(s^{2}+20 s+100\right)
\end{aligned}
$$


for $h_{11}=-2 h_{12}$, and

$$
\begin{aligned}
-2 h_{21} & =-20 \\
\left(2 h_{11}-1\right)\left(h_{21}-2 h_{22}-1\right)-4 h_{12} h_{21} & =-100 .
\end{aligned}
$$

Solving (45), we obtain (for example)

$h_{11}=5.5, \quad h_{12}=-2.25, \quad h_{21}=10, \quad h_{22}=-9.5$

Step 5. In this case, from (25c) we have

$$
\begin{aligned}
\bar{f}_{2}\left(\bar{x}_{1}, \bar{x}_{2}, u\right) & =f_{2}\left(\bar{x}_{1}, \bar{x}_{2}, u\right)-E_{21} \frac{\mathrm{d}^{\alpha} y}{\mathrm{~d} t^{\alpha}}+A_{21} y \\
& =\left[\begin{array}{c}
x_{1} x_{2}+x_{3}^{2}+x_{2} u \\
x_{2} x_{3}+2 x_{1} u^{2}
\end{array}\right], \\
\bar{y} & =E_{11} \frac{d^{\alpha} y}{\mathrm{~d} t^{\alpha}}+A_{11} y-f_{1}\left(\bar{x}_{1}, u\right) \\
& =\left[\begin{array}{c}
x_{2}^{2}+u^{2} \\
x_{2} x_{4}+2 u
\end{array}\right] .
\end{aligned}
$$

The desired reduced-order fractional observer of the system is described by the equation

$$
\begin{aligned}
{\left[\begin{array}{ll}
0 & 1 \\
1 & 0
\end{array}\right] \frac{\mathrm{d}^{\alpha} \hat{x}_{2}}{\mathrm{~d} t^{\alpha}}=} & {\left[\begin{array}{cc}
-10 & 0 \\
20 & 10
\end{array}\right] \hat{x}_{2} } \\
& -\left[\begin{array}{c}
x_{1} x_{2}+x_{3}^{2}+x_{2} u \\
x_{2} x_{3}+2 x_{1} u^{2}
\end{array}\right] \\
& -\left[\begin{array}{cc}
455 & -2.75 \\
10 & -9.5
\end{array}\right] \bar{y}
\end{aligned}
$$

or

$$
\begin{aligned}
\frac{\mathrm{d}^{\alpha} \hat{x}_{2}}{\mathrm{~d} t^{\alpha}}= & {\left[\begin{array}{cc}
-20 & 10 \\
-10 & 0
\end{array}\right] \hat{x}_{2} } \\
& -\left[\begin{array}{c}
x_{1} x_{2}+x_{3}^{2}+x_{2} u \\
x_{2} x_{3}+2 x_{1} u^{2}
\end{array}\right] \\
& -\left[\begin{array}{cc}
10 & -9.5 \\
5.5 & -2.75
\end{array}\right] \bar{y} .
\end{aligned}
$$

\section{Concluding remarks}

Fractional descriptor reduced-order nonlinear observers for a class of fractional descriptor continuous-time nonlinear systems have been proposed. A design procedure for fractional descriptor observers has been proposed and illustrated on a numerical example.

The discussion can be easily extended to perfect fractional descriptor reduced-order observers and fractional descriptor discrete-time linear systems. An open problem is extension to fractional descriptor 2D continuous-discrete nonlinear systems.

\section{Acknowledgment}

This work was supported by the National Science Centre in Poland under the work no. 2014/13/B/ST7/03467.

\section{References}

Cuihong, W. (2012). New delay-dependent stability criteria for descriptor systems with interval time delay, Asian Journal of Control 14(1): 197-206.

Dodig, M. and Stosic, M. (2009). Singular systems state feedbacks problems, Linear Algebra and Its Applications 431(8): 1267-1292.

Dai, L. (1989). Singular Control Systems, Lecture Notes in Control and Information Sciences, Vol. 118, Springer-Verlag, Berlin.

Fahmy, M.M. and O'Reill, J. (1989). Matrix pencil of closed-loop descriptor systems: Infinite-eigenvalue assignment, International Journal of Control 49(4): 1421-1431.

Gantmacher, F.R. (1960). The Theory of Matrices, Chelsea Publishing Co., New York, NY.

Guang-ren, D. (2010). Analysis and Design of Descriptor Linear Systems, Springer, New York, NY.

Kaczorek, T. (1992). Linear Control Systems, Vol. 1, Research Studies Press, J. Wiley, New York, NY.

Kaczorek, T. (2001). Full-order perfect observers for continuous-time linear systems, Bulletin of the Polish Academy of Sciences: Technical Sciences 49(4).

Kaczorek, T. (2004). Infinite eigenvalue assignment by an output feedback for singular systems, International Journal of Applied Mathematics and Computer Science 14(1): 19-23.

Kaczorek, T. (2008). Fractional positive continuous-time linear systems and their reachability, International Journal of Applied Mathematics and Computer Science 18(2): 223-228, DOI: 10.2478/v10006-008-0020-0.

Kaczorek, T. (2011a). Positive linear systems consisting of $n$ subsystems with different fractional orders, IEEE Transactions on Circuits and Systems 58(7): 1203-1210.

Kaczorek, T. (2011b). Selected Problems of Fractional Systems Theory, Springer-Verlag, Berlin.

Kaczorek, T. (2012a). Checking of the positivity of descriptor linear systems with singular pencils, Archive of Control Sciences 22(1): 77-86.

Kaczorek, T. (2012b). Positive fractional continuous-time linear systems with singular pencils, Bulletin of the Polish Academy of Sciences: Technical Sciences 60(1): 9-12.

Kaczorek, T. (2013). Descriptor fractional linear systems with regular pencils, Asian Journal of Control 15(4): 1051-1064.

Kaczorek, T. (2014a). Fractional descriptor observers for fractional descriptor continuous-time linear system, Archives of Control Sciences 24(1): 5-15.

Kaczorek, T. (2014b). Reduced-order fractional descriptor observers for fractional descriptor continuous-time linear systems, Bulletin of the Polish Academy of Sciences: Technical Sciences 62(4): 889-895.

Kaczorek, T. (2015). Prefect observers of fractional descriptor continuous-time linear systems, in K.J. Latawiec et al. 
(Eds.), Advances in Modeling and Control of Non-integer orders Systems, Lecture Notes in Electrical Engineering, Vol. 320, Springer, Berlin/Heidelberg, pp. 5-12.

Kociszewski, R. (2013). Observer synthesis for linear discrete-time systems with different fractional orders, Pomiary Automatyka Robotyka (2): 376-381, (on CD-ROM).

Kucera, V. and Zagalak, P. (1988). Fundamental theorem of state feedback for singular systems, Automatica 24(5): 653-658.

Lewis, F.L. (1983). Descriptor systems, expanded descriptor equation and Markov parameters, IEEE Transactions on Automatic Control AC-28(5): 623-627.

Luenberger, D.G. (1977). Dynamical equations in descriptor form, IEEE Transactions on Automatic Control AC-22(3): 312-321.

Luenberger, D.G. (1978). Time-invariant descriptor systems, $A u$ tomatica 14(5): 473-480.

Matignon, D. (1996). Stability result on fractional differential equations with applications to control processing, IMACSSMC Proceedings, Lille, France, pp. 963-968.

N'Doye I., Darouach M., Voos H. and Zasadzinski M. (2013). Design of unknown input fractional-order observers for fractional-order systems, International Journal of Applied Mathematics and Computer Science 23(3): 491-500, DOI: 10.2478/amcs-2013-0037.

Oldham, K.B. and Spanier, J. (1974). The Fractional Calculus, Academic Press, New York, NY.

Ostalczyk, P. (2008). Epitome of the Fractional Calculus: Theory and Its Applications in Automatics, Technical University of Łódź Press, Łódź, (in Polish).

Podlubny, I. (1999). Fractional Differential Equations, Academic Press, New York, NY.
Van Dooren, P. (1979). The computation of Kronecker's canonical form of a singular pencil, Linear Algebra and Its Applications 27: 103-140.

Vinagre, B.M., Monje, C.A. and Calderon, A.J. (2002). Fractional order systems and fractional order control actions, Lecture 3, IEEE CDC'02, Las Vegas, NV, USA.

Virnik, E. (2008). Stability analysis of positive descriptor systems, Linear Algebra and Its Applications 429: 2640-2659.

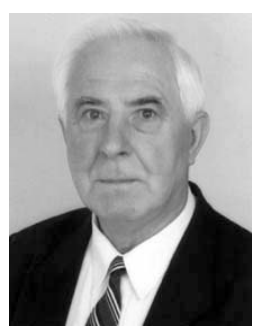

Tadeusz Kaczorek received the M.Sc., Ph.D. and D.Sc. degrees in electrical engineering from the Warsaw University of Technology in 1956, 1962 and 1964, respectively. In the years 1968-69 he was the dean of the Electrical Engineering Faculty, and in the period of 1970-73 he was a deputy rector of the Warsaw University of Technology. In 1971 he became a professor and in 1974 a full professor at the same university. Since 2003 he has been a professor at the Białystok University of Technology. In 1986 he was elected a corresponding member and in 1996 a full member of the Polish Academy of Sciences. In the years 1988-1991 he was the director of the Research Center of the Polish Academy of Sciences in Rome. In 2004 he was elected an honorary member of the Hungarian Academy of Sciences. He was granted honorary doctorates by 13 universities. His research interests cover systems theory, especially singular multidimensional systems, positive multidimensional systems, singular positive 1D and 2D systems, as well as positive fractional $1 \mathrm{D}$ and $2 \mathrm{D}$ systems. He initiated research in the field of singular 2D, positive 2D and positive fractional linear systems. He published 28 books ( 8 in English) and over 1100 scientific papers. He also supervised $69 \mathrm{Ph} . \mathrm{D}$. theses. He is the editor-in-chief of the Bulletin of the Polish Academy of Sciences: Technical Sciences and a member of editorial boards of ten international journals.

Received: 19 January 2015 Revised: 10 March 2015 Accepted: 10 June 2015 